\title{
The Effect of Realistic Appearance of Virtual Characters in Immersive Environments - Does the Character's Personality Play a Role?
}

\author{
Katja Zibrek, Elena Kokkinara and Rachel McDonnell
}
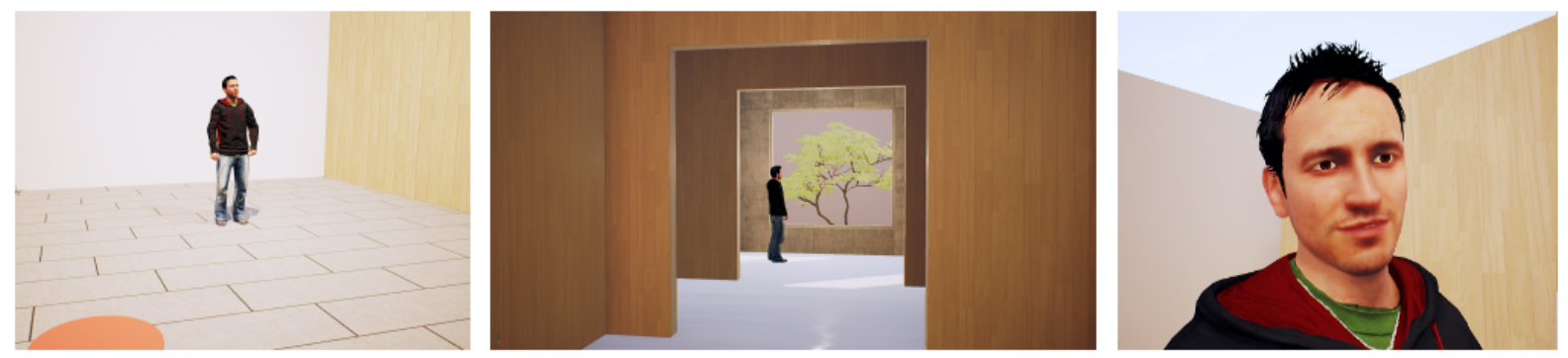

Fig. 1. Screenshots of the virtual environment and realistic character used in our experiment.

\begin{abstract}
-
Virtual characters that appear almost photo-realistic have been shown to induce negative responses from viewers in traditional media, such as film and video games. This effect, described as the uncanny valley, is the reason why realism is often avoided when the aim is to create an appealing virtual character. In Virtual Reality, there have been few attempts to investigate this phenomenon and the implications of rendering virtual characters with high levels of realism on user enjoyment. In this paper, we conducted a large-scale experiment on over one thousand members of the public in order to gather information on how virtual characters are perceived in interactive virtual reality games. We were particularly interested in whether different render styles (realistic, cartoon, etc.) would directly influence appeal, or if a character's personality was the most important indicator of appeal. We used a number of perceptual metrics such as subjective ratings, proximity, and attribution bias in order to test our hypothesis. Our main result shows that affinity towards virtual characters is a complex interaction between the character's appearance and personality, and that realism is in fact a positive choice for virtual characters in virtual reality.
\end{abstract}

Index Terms-Personality, virtual characters, virtual reality, perception

\section{INTRODUCTION}

Virtual reality, in which people have particular or all their senses completely immersed in an artificially created world, is becoming important in areas such as education, medicine, entertainment, etc. Currently, major corporations such as Facebook, are investigating the efficacy of communication between avatars in virtual reality, since users will be able to modify the appearance of their own self-representations. However, the appearance of an avatar could influence the way a message is communicated: the seriousness of an emotional situation, for example, could be assessed as unimportant simply because it is communicated with an unrealistic virtual character. This could have crucial consequences in applications, such as medicine and training, where the accuracy of transmitted information is vital.

When addressing the question of how the appearance of the virtual character influences one's perception, the most common concern is that of visual realism. The uncanny valley, proposed by Mori [33], describes a negative response from the user who is observing a near photo-realistic character. While the existence of the uncanny valley is still an ongoing investigation, the most common problem is the variety of stimuli used to represent increasing levels of realism (see,

\footnotetext{
- Katja Zibrek, Elena Kokkinara and Rachel McDonnell are with the Graphics, Vision, and Visualisation Group at Trinity College Dublin. E-mail:zibrekka@tcd.ie,ramcdonn@tcd.ie,redarahel@gmail.com.
}

Manuscript received xx xxx. 201x; accepted xx xxx. 201x. Date of Publication xx xxx. 201x; date of current version xx xxx. 201x. For information on obtaining reprints of this article, please send e-mail to: reprints@ieee.org. Digital Object Identifier: $x x . x x x x / T V C G .201 x . x x x x x x x$ e.g., Katsyri et al. [27]). In our research, we approached this problem by designing levels of visual realism of a character by changing its render style ${ }^{1}$, while keeping the realism of motion and shape constant.

The second concern is - does realism matter in an immersive environment? Previous studies have put more importance on matching behavior and appearance of the character (see, e.g., Bailenson et al. [4]), but proposed that the interaction between behaviour and appearance is complex. To study one such behavior which is commonly experienced in real life interactions with people, we designed characters, who exhibit personality traits. We expected that the type of personality would interact with the character's appearance, where negative personalities might either lower the appeal of unpleasant looking characters, or might actually make them more appealing due to the match between appearance and expected behaviours. The novelty of our study is therefore, that we looked at complex but naturally present behavior when observing other people (personality traits), and we limited the concept of realism to only changing the render style of the character, without modifying the actual geometry or motion of the virtual character's model.

To establish a possible relationship between personality and render style in virtual reality and how it mediates the response towards virtual characters, we created an immersive, game-like scenario experiment, and displayed it to members of the general public, visitors to a Science Gallery Dublin ${ }^{2}$ exhibition. Because of this, we were able to collect

\footnotetext{
${ }^{1}$ The term render style describes visual qualities of the character other than shape; including material properties, lighting setup and post-processing effects.

${ }^{2}$ Science Gallery is an international chain of art exhibition centres with a science outreach.
} 
responses on an exceptionally large sample (1106 individuals). This study provides a valuable insight into how the character's appearance influences our response to virtual characters and the role the character's personality plays in perception. Our contribution to the field extends the question in an engaging, virtual reality environment, and provides guidelines for this developing platform.

\section{BACKGROUND}

Continuing from Mori's [33] early identification of the "uncanny valley", a substantial amount of research focused on discovering how visual realism could induce a negative response in the viewer (see Katsyiri et al. [27] for an overview). Many studies put emphasis on the evidence that the uncanny valley is a result of the mismatch in realism between elements of character design. MacDorman et al. [30] investigated this by mismatching realism of texture and model geometry, while Yamada et al. [45], and Seyama et al. [39] focused on mismatching the stimuli from separate categories (human with non-human faces) Motion, or the mismatch between realism and appearance and motion, is typically associated with the uncanny effect, since biological motion is a very strong cue by itself, as demonstrated by Johansson et al. [25], and another evidence presented by Chaminade et al. [9] showed motion is more inaccurately perceived when the realism of the model increases. Certain areas, such as the face, are particularly vulnerable for the perceived unpleasantness when moving, as studies of Hodgins et al. [23] and Tinwell et al. [41] showed. Another study by Zell et al. [46] analysed the effect of visual realism further by separating the realism of shape and material. Material was found to be the main predictor of appeal (e.g., blemish-free skin is most appealing) and shape the dominant predictor of realism (e.g., exaggerated features of the character are common in cartoons, but not typical for real humans). These studies show that while increasing visual realism could expose the character to a harsher judgement, realism itself can be created in different ways and is by itself not necessarily a predictor of affinity towards the character. Indeed, changing render style without the changes to the mesh geometry of the character can change appeal ratings regardless of the perceived realism as some studies of McDonnell et al. [31] and Carter et al. [8] show. However, realism of characters has other perceptual effects, such as how trustworthy people find the character [31], which information they disclose to it, as found by Ring et al. [37], and even how they empathise with it [42]. Wallraven et al. [43] found also, that render style can affect emotion recognition and intensity.

Personality refers to individual differences in characteristic patterns of thinking, feeling and behaving. Due to its continuous examination and re-evaluation, the "Big Five" theory (see for example Goldberg [20], Costa and McCrae [11], and John et al. [26]) is perceived by many to be the standard description of human personality. The Big Five is a hierarchical model of personality traits with five broad factors (extraversion, agreeableness, conscientiousness, openness to experience, and emotional stability). Each bipolar factor (e.g., extraversion vs. introversion) is further described by specific facets and traits (e.g. extraverts are talkative, sociable, but people with low emotional stability are seen as anxious and easily upset). A particular study by Musek [34] showed that the facets of the Big Five, could be summarised as desirable (having high scores on all the facets) vs. less desirable (low on these facets) blend of all socially valued traits. There have also been attempts to systematically study personality expressed by virtual characters. Tinwell et al. [41] showed that inaccurate motion in the upper facial region is associated with attributing psychopathic traits to the character. Hyde et al. [24] found evidence that speed of body motion is positively correlated with the perceived extraversion of the character. The study of Zibrek et al. [48] showed a possible link between appearance and personality, where the character with a less appealing style was attributed less desirable traits (lower conscientiousness) than the other, more appealing style. However, the study only investigated two styles and was conducted on-screen, as opposed to being presented in virtual reality, which is the focus of our current study.

When observing real people, inconsistencies or deviations from normality can impede the ease of cognitive processing and this can be detected with the measure of attribution bias used by Gilbert et al. [16]. This error is the tendency to associate behaviour to person's stable personality traits rather than exceptional situational factors which could provoke the person's behaviour [28]. Similarly, Cheetham and Jancke [10] proposed that low-level processing, i.e. cognitive difficulty, was involved in inducing a negative response when observing virtual characters. Based on this assumption, the uncanny valley could be related to cognitive difficulty and on a response level, the answer could reflect the fundamental attribution error (for more information on attribution error, see Ross [38]).

In immersive virtual environments (IVE's, as described by Bailenson et al. [3]), the response to virtual characters can be successfully analysed with behavioral measures. Proximity is one of the measures which focuses on measuring the minimum distance of approaching the character in VR and has been commonly used to study interpersonal relations observed in real human interactions, such as engagement (see Blascovich et al. [6]) and social status (see Latta [29]), but proximity has also been established as a measure of co-presence with the virtual character (work of Bailenson and colleagues [2-4]). The usual setup includes a task that prompts participants to move close to the virtual character, presented in virtual space. While participants are moving, their trajectory is accurately recorded and minimal front distance is used to asses the level of comfort people take when approaching a virtual human - further distance from the character indicates higher co-presence. The relation between appearance and proximity gave conflicting evidence, where appearance realism had no effect on the viewer's response, shown by Slater et al. [40] and Garau et al. [15] but increasing the anthropomorphism levels was positively correlated with co-presence, as shown by Nowak et al. [36]. However, a common result suggests that a mismatch between the realism of behaviour and appearance lowers the feeling of co-presence [4]. A new study by Zibrek et al. [47], comparing the effect of render style and agency in virtual reality, found that realism was related to higher levels of appeal but did not influence the proximity measure. The mentioned study, however, only used characters performing exercising motions, with minimal facial animation and interactive cues, which could have been a reason for relatively low co-presence.

Our current study was designed to answer the question about the importance of realism and the role of personality traits in the overall perception of this character. We are particularly interested to know, if the responses associated with the uncanny valley exist in virtual reality as well. To test this, we used a diverse methodology, consisting of subjective ratings, low-level perception and behavioral responses described below.

\section{Stimuli Creation \\ 3.1 Render Styles}

The stimuli creation and experiment design is similar to the experiment described in Zibrek et al. [47] with a few changes. For the stimuli, we used five different render styles, shown in Figure 2. We chose these styles to have three examples of appealing render styles while the two obviously eerie render styles served as a reliability measure - we predicted these styles would increase the level of eeriness, lower appeal and increase personal distance between them and participants. The reason behind using intentionally creepy render styles was to induce a more extreme reaction from the participant to compare with the reaction towards the response to the realistic style. The characters were created in Autodesk 3ds Max 2015® and finalised in Unreal Engine 4.9. ( (UE4). We chose render styles which were rated appealing but which varied in realism, as described in the study of Zibrek et al. [47]: Realistic style, which represented the highest level of realism and had the most complex material structure with subsurface scattering for skin, refraction for eyes and transparency for hair. Toon $C G$ was a less realistic, cartoon style character, and Toon Shaded was the least realistic character, rendered with a Cel shading post-process effect in UE4. The additional unappealing Creepy render style was designed to induce a sense of discomfort by mismatching realistic appearance with a creepy component - grossly enlarged eyes, to induce the uncanny effect (see Seyama et al. [39], and Tinwell et al. [41]). For this render style, only simple maps of the Realistic render style were used (texture, normal 
and specular map) and subsurface scattering effect was removed. In addition, the mesh of the virtual character was edited in 3Ds Max 2015 to enlarge the eyes out of proportion. Zombie render style was created to be obviously less realistic and unappealing, by additionally changing the diffuse map for the skin and the eyes of the Creepy render style by adding an unnatural colour (green for skin and red for the eyes). These changes resulted in an appearance of a "zombie" character, seen in movies and games.

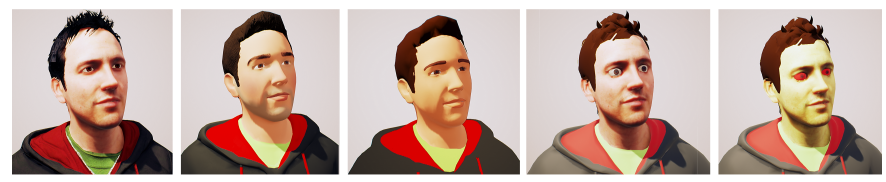

Fig. 2. Render styles of the character used in the experiment (from left to right): Realistic, Toon CG, Toon Shaded, Creepy and Zombie render style.

For the creation of the animations, we used previously captured body and facial motion from a professional actor [48], where a 21-camera Vicon optical system was used, and a Behringer C-2 studio condenser microphone was placed in front of the actor to record sound. The captured body and facial motions (without finger or eye movement) and sound were then imported separately to UE4.

\subsection{Personality Situations}

The actor was instructed to react to different emotional situations which we created in order to bring out the Big Five traits and their polar opposites. The personality descriptions are taken from the 10 situations featured in the study of Zibrek et al. [48], which were designed based on the available operationalisations of personality from the existing literature, such as descriptions of the Big Five facets (in studies of Goldberg [20], Costa and McCrae [11], John et al. [26]), zero-acquaintance observations (Mehl et al. [32]) and language analysis (Gill and Oberlander [18], Dewaele and Furnham [13]). For example, the actor was told he would portray an extraverted personality in a situation where he received a new television as a birthday present from his friends. He was instructed to express gratitude, be humorous, show positive emotions and mention how important his friends are. He was also given information on the type of body language (e.g., expressive, physically animated) and language (e.g., informal, use plural "we") he should use. The length of each performance depended on the actor's free interpretation of the character and the capture was stopped when all the main characteristics of the personality were expressed. On average, the situations were 30 seconds long. We only used a subset of personality situations, which were suitable for the investigation of empathy and low-level perception (attribution bias, proximity and co-presence): Agreeable/Non Agreeable, Extraverted/Introverted and Emotionally Stable/Neurotic represented suitable situations as well as enough variation to our stimuli. We also renamed the negative poles (Low Agreeable Personality into Non Agreeable, Low Extraverted Personality into Introverted, and Low Emotionally Stable Personality into Neurotic) for practical purposes since the clear distinction that we were expecting low ratings on a particular TIPI scale for a portrayed personality was not needed here.

\subsection{Virtual Environment}

The virtual scene was realised using UE4 and consisted of a "waiting room", "training" and "experiment room". The waiting room was designed to engage participants in discovering the environment, therefore we used aesthetic design, footsteps sounds, and atmosphere music playing in the background. This room also featured the Realistic character, with only idle motion applied (blinks, slight sways in the hips when changing the resting leg). This character was purposefully presented in this room to anchor participants' responses to the render style they would encounter in the experiment condition. More specifically, if they were randomly assigned the least realistic render style (Toon Shaded) in the experiment room, they would know that this is not as realistic as the character could be, since they saw a more realistic representation before. To ensure all participants would come close enough to the character to see the details, we used a collider around it as a trigger for unlocking the experiment room. When participant's view-camera would intersect the collider of $1.5 \mathrm{~m}$ radius surrounding the character, this would trigger the door access to the experiment room. The door opening event would then similarly be triggered when the view-camera was in the vicinity of the door.

The part of the environment where the experiment started, was a simplistic space with two virtual rooms: one for the training session and the other for the experiment session. The first room contained a box and the other a character in one randomly chosen render style (see Figure 3), and a red spot on the floor, which was placed $4 m$ meters away from the virtual character. This position was chosen as an optimal observation distance, that would give some personal space to the viewer when watching the character speak, but at the same time details of the character could still be visible. For the training, the box was chosen to estimate the observation distance participants took when reading the word, and therefore served as the control condition.

We chose a simplistic environment setup with no decoration, in order to avoid distraction of participants view from the virtual characters. The lighting consisted of a general "sky light" and a two point lights directed towards the character, casting shadows. No music was playing in the background in the experiment room, as the participants were instructed to listen carefully to the character's speech.
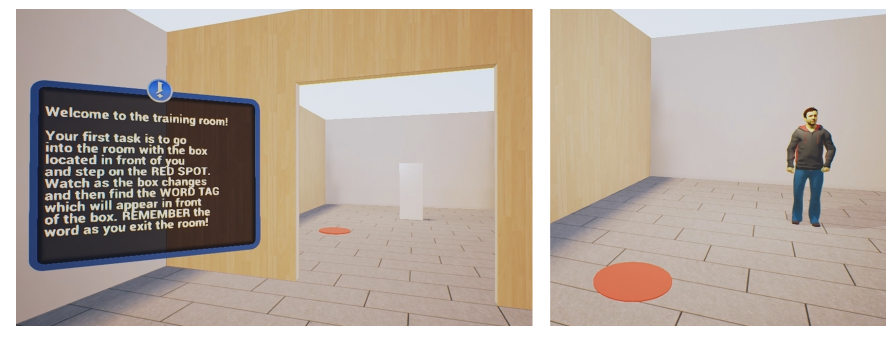

Fig. 3. Left: an example of the information box with guidelines for the participant before the training room task. The box was presented as a blue dot in the environment and the text box only expanded when participant moved closer to it. Right: experiment room example.

We created a first person camera view which tracked the movement of the participant. Participants were able to navigate (translation and rotation of the camera) using an Xbox controller. In the virtual room, we placed a red spot which served as the observation distance for the proximity measure. Access closer than $\sim 4$ meters to the character was initially restricted with an invisible sphere collider that was placed around the character. Once the first person camera was navigated to the red spot, the camera translation was locked for the duration of the character's speech. Participants were only allowed to approach the character after it finished talking.

A notable characteristic of our experiment was also that it was programmed to run automatically from start to finish, similar to a gamelike scenario, with all instructions and the tasks being presented in the environment. This allowed the participant to move through the experiment independently, without asking questions in the middle of the experiment or even taking off their headset as this would break the immersion. We also expected that participants would be motivated and dedicate enough time to our exhibit if it was built like a video game. Making the experiment run in this way was a demanding task which required extensive pre-testing to ensure the participants would not be stuck in one area and not know how to progress with the tasks. Some guides were implemented as colliders which prevented the motion of the participant to continue, e.g. through the window. The presentation of tasks however, was implemented in a form of pop-up information text boards (Figure 3), which triggered when the participant came close to a blue information symbol in the environment. All data was saved at the end of the experiment. 


\section{EXPERIMENT Design}

\subsection{Experiment Apparatus and Protocol}

This experiment was featured at the Science Gallery Dublin exhibition, which was running continuously for three months in 2016. The exhibit included a monitor, Xbox controller, headphones, Oculus DK2, and a rotating chair (sample picture of the setup is shown on Figure 4). Visitors could participate in the experiment voluntarily. They were introduced to the task with the help of the mediator employed by the gallery who was trained to follow the experiment protocol. The protocol, included important points about hardware maintenance, security issues, and instructions regarding working with participants. For example, no children under 13 years of age were allowed, participants over 13 but under 18 years of age were informed their data would not be collected or included in the analysis, visitors who had trouble understanding English language needed to have a translator; if no translator was present, they could not continue with the experiment. In case of nausea, the experiment had to be terminated and the participants remained seated for a couple of minutes and were given a glass of water.

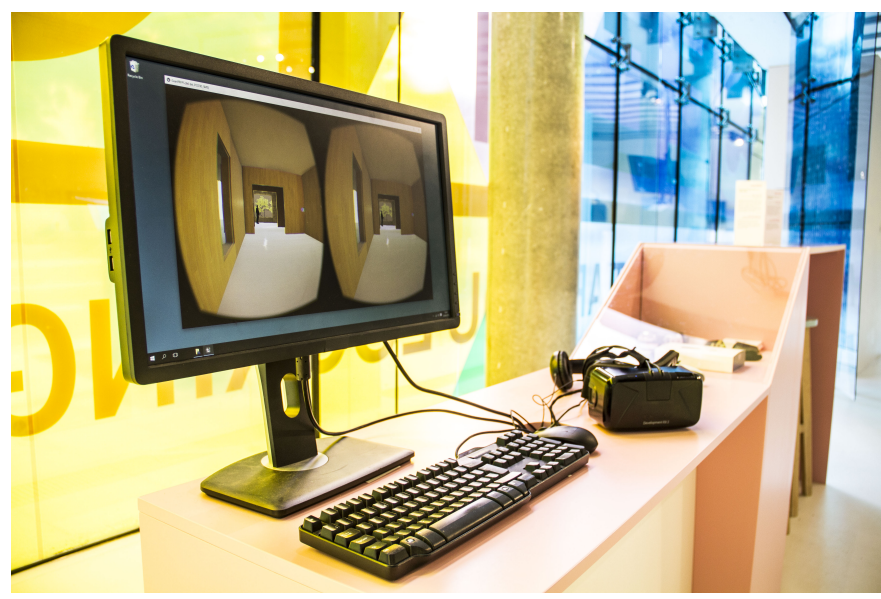

Fig. 4. The setup at the Science Gallery exhibition. Participants for this experiment were visitors of the gallery who volunteered to participate.

\subsection{Participants and Procedure}

Due to an overwhelming response from the visitors of the Science Gallery, we were able to collect data from over 2000 participants, from which 1106 (429 females, 677 males; average age: 28.7 ) were included in the analysis. The other participants were excluded due to failing to fill out the demographics, have taken the experiment before, or gave inaccurate responses to the memory task which was a way of testing if participants payed attention. University department and Science Gallery committee ethics approval was secured for the experiment and participants were invited to read and agree to the approved consent form before starting the experiment.

We also programmed the experiment in such a way, that the subjective responses from the participants were saved only if they finished the whole experiment. This way, if someone decided to leave the experiment before finishing it, their data was not included in the analysis. All participants had normal or corrected to normal vision.

The participants were asked to sit in front of the monitor where they were first presented with an electronic consent form and information sheet. They then filled out the demographics: age, sex, game experience, experience with virtual characters, native English speaker or not and a question if they have taken the experiment before. With the help of the mediator, they were given the headset and a set of headphones to put on their head.

The experiment had three stages: waiting room, where the participant would get used to the environment and controls, training and experiment room.

\subsubsection{Waiting Room}

By entering this room, the instruction box, in the form of a pop-up window (Figure 3) appeared in front of the participant's view-camera which described the use of controls. After, the participant was free to move and explore the room. Other information boxes guided the participant to the first task, which was to go closer to the realistic virtual character which was standing near the window (Figure 1, second image). After they got close enough to the realistic character (triggered by contact of the view-camera's and the character's collider), the next level unlocked, which was accessible to the door with a sign "Experiment Room". The door opened when they navigated close to it, so that the door collider was intersected with the view-camera, and this action teleported them to the next level, where they were presented with the next two rooms - training and experiment. The experiment room only unlocked when the training room was completed.

\subsubsection{Training Room}

The instructions guided the participant to enter the training room and when they reached the red spot (Figure 3) they were locked in position and were instructed to observe the training example, which was a box, changing colours for a dedicated amount of time, after which instructions to go closer and find the word tag appeared. When moving closer and away from the box, the position trajectory of the first person camera was recorded. After exiting the room, a multiple choice questionnaire appeared on the virtual board in front of them, asking them to choose the word tag they found in the room.

\subsubsection{Experiment Room}

After, the experiment room unlocked and they were able to walk towards the red spot in this room, and after locking the position, observed the character in one randomly assigned render style, expressing one randomly ${ }^{3}$ chosen personality (set of 6 personalities). After the animation stopped playing, they had the same task to memorise the word tag and exit the room. Upon exiting, the questionnaire board appeared with the empathy, uncanny valley and co-presence questions. After they answered all the questions, the experiment self-terminated.

\subsection{Analysis}

In this research, we used a combination of three different measures: subjective responses, an implicit measure and a behavioral measure. For subjective responses, we designed a questionnaire, which was put on a virtual board in the environment. The questions (apart from the first one) were rated on a 5-point Likert scale, ranging from 1-Not at all to 5-Extremely. Table 1 shows the questions used in the experiment.

The first question was designed to measure attribution bias, which requires the participant to make a decision about the locus of the character's reaction to the situation. We constructed this question similarly to the study of Gilbert et al. [17] after observing the character portraying a personality. We posed the following question:

- "You witnessed the character reacting to a situation. In your opinion, what is the MAIN cause of the character's behaviour? ( 1 - character's unique way of behaving; 2 - situation made the character behave in this way)":

$\begin{array}{cc}1 & 2 \\ \text { Character } & \text { Situation }\end{array}$

The next three questions were related to the measures of empathy Concerned, Excited and Uneasy. They were based on the understanding of empathy as experiencing the emotions of others, with the Concerned scale taken from the study of Davis et al. [12], Excited, which is an emotion of positive valence signaling engagement with the character, taken from the PANAS questionnaire [44] and a negative emotion taken

\footnotetext{
${ }^{3}$ We removed the randomness towards the end of the exhibition to achieve a more counterbalanced data set.
} 
Table 1. Likert-scale subjective responses to explore the effect of render style on the perception of virtual characters.

\begin{tabular}{|c|c|c|}
\hline Group & Question & Statement \\
\hline \multirow{3}{*}{ Empathy } & Concerned & "I feel concerned about the character:" \\
\hline & Excited & "Ifeel excited after watching the character:" \\
\hline & Uneasy & "I feel uneasy after watching the character:" \\
\hline \multirow{3}{*}{ Realism } & Appearance Realism & $\begin{array}{l}\text { "I found the character's appearance realistic } \\
\text { ("Not at all" = the character's appearance is highly } \\
\text { stylised like in cartoons, "Extremely" = it could almost } \\
\text { be mistaken for a photograph)." }\end{array}$ \\
\hline & Movement Realism & "I found the character's movements realistic." \\
\hline & Overall Realism & "I found the character realistic overall." \\
\hline \multirow{3}{*}{ Affinity } & Appeal & $\begin{array}{l}\text { "I found the character appealing } \\
\text { ("Extremely" = the character is one that you } \\
\text { would like to watch more of and would be } \\
\text { captivated by a game with that character as the lead)". }\end{array}$ \\
\hline & Eerie & $\begin{array}{l}\text { "I found the character eerie } \\
\text { ("Not at all" = character restores a sense of } \\
\text { security, confidence, calm in me. } \\
\text { "Extremely" = character is gloomy and leaves } \\
\text { me with a sense of fear)." }\end{array}$ \\
\hline & Familiar & $\begin{array}{l}\text { "I found the character familiar } \\
\text { ("Extremely" = I have seen something } \\
\text { similar to it before)." }\end{array}$ \\
\hline Co-presence & Co-presence & $\begin{array}{l}\text { "I perceived that I was in the presence } \\
\text { of another person in the virtual room with me." }\end{array}$ \\
\hline
\end{tabular}

from CAM battery [19]: Uneasy. The questions which follow are the uncanny valley questions (Affinity and Realism groups, see Table 1), and are based on measures previously used by McDonnell et al. [31]. The co-presence question is taken from Bailenson et al. [3].

For the behavioural measure, we used proximity, which is the minimum distance a person takes to the character in order to perform a specific task, in our case the word tag near the character. This task is similar to the design used by Bailenson and colleagues [3], except that the word was simple to remember ("table", "chair"), since the participants had to memorise it and report it later (design used in Zibrek et al. [47]). The participant was first presented with the floating text in front of a training box, and had to leave the observation point (red spot) to find the word. The position of the first person view-camera was tracked and the minimum distance from the box was an estimate of the distance from where participant could read the word. The minimum distance when memorising the word tag in front of the character in the experiment room was then used for the proximity measure.

\subsubsection{Statistical Analysis}

The data collected for a particular render style and personality combination were almost counterbalanced. For the Realistic style, we collected data from 222 participants, where the average number of participants per personality $\left(n_{p}\right)$ was $37(S D=4.7)$. Other styles: Toon $\mathrm{CG}=227$ $\left(n_{p}: 37.8, S D=10\right)$, Toon Shaded $=227\left(n_{p}: 37.8, S D=2.3\right)$, Creepy $=212\left(n_{p}: 35.3, S D=6.6\right)$, Zombie $=218\left(n_{p}: 36.3, S D=3.4\right)$.

To explore the most robust effects of Render Style on people's perception, we conducted a two-way ANalysis Of VAriance (ANOVA) with between-subject factors Render Style (5 examples) and Personality (6 examples) for every dependent variable (subjective responses and Proximity). In addition, we analyse the effect of Render Style individually inside each Personality. We did so by using one-way ANOVA with between-subject factor Render Style.

ANOVA is considered robust for the deviations from normality. However, we used Levene's test to check for homogeneity of variance and for variables, where the assumption was breached, we did a log transformation on the data and repeated the ANOVA process on the transformed data. Tukey Honestly Significant Difference (HSD) test was used for post-hoc comparisons.

For Proximity, the minimum distance, expressed as Euclidian distance of the current camera (user) position and the virtual character position in the virtual space, was calculated.

Correlations between Proximity and questionnaire items were calculated using Sperman's Rank Order test, since we found the Proximity variable was not normally distributed (Kolmogorow-Smirnov test).

To analyse attribution bias, we explored if the categorisation of character's behaviour to either Character or Situation was significantly different according to factors Personality and Render Style. We approached this by using crosstabulations and computed significant differences in attribution bias across Render Style and Personality using Pearson Chi-Squared test. Post-hoc was conducted by partitioning the Chi-Squared test into independent 2-way component tables.

We also conducted a demographics analysis, where we were interested in the effects of Sex, Age, Native (if the participant is a native English speaker or not), Game Experience and Character Experience. We conducted a generalized linear model analysis to screen for possible effects on our data (see Table 2, in Supplementary Material). This analysis is a form of linear regression, which is robust for violations of normality and finds systematic effects that can be made linear through a suitable transformation [35]. We chose this model because it can predict effects of multiple variables on our data without specific requirements for normality or homoscedasticity of variance.

All main effect and post-hoc tests are reported in Table 1 in the Supplementary Material.

\section{Results}

We present the analysis of results in separate sections, immediately followed by the discussion, for clarity purposes:

Section a) where we report overall main effects of Render Style and its interaction with Personality on the dependent variables.

Section $b$ ) where we analyse the effect of Personality on the dependant variables. We continue to analyse the effects of Render Style separately inside each Personality.

Section c) where we report the results of the dependent variable Attribution. Here, we analyse significant differences in how participants perceived the behaviour of the character - as coming from his own personality or as a result of the situation.

Section $d$ ) where we provide additional information on how some aspects of participants could influence our data. We did not include participant sex or other demographic information into the main analysis since we had a large number of variables and potential effects. Therefore, we analyse potential effects of demographic variables by using generalised linear models to discuss possible effects on the results we have collected in our study.

\section{1 a) The effect of Render Style}

When conducting the overall analysis of main effects for Render Style, not many variables were affected (for values, see Supplementary Material, Table 1), even the perceived realism was rated similarly for different render styles, as the graph in Figure 5 shows. Significant difference in overall realism was found only between Realistic and the least realistic, Toon Shaded render style. Also, closer proximity towards the Zombie render style was unexpected, since participants should keep further away from an intentionally uncanny character. It is possible participants found the character interesting and came closer to investigate details, and, on the other hand, also show a possible lack of interest for the Toon CG render style. Perhaps Toon CG render style does not have interesting details on its texture and no visible (or intentional) flaws. In addition to not finding any correlations between proximity and other responses from the participant, these results could indicate that our measure of proximity was not related to how comfortable people were to getting close to the character but rather served the examination of details of the render style. This is perhaps evident also from the result that participants stayed significantly further away from the box in the training condition (Training minimum distance: $M=200.97 \mathrm{~cm}, S D=124.03$; Experiment minimum distance: $M=173.57, S D=99.84 ; t(2210)=5.72, p \approx 0)$, when completing the label task. It seems they did not approach the box closer because they were not interested in observing it.

Interactions were found for Eerie and Co-presence variables as well, but the results are hard to interpret (see Supplementary Material, Table 1). A higher co-presence for extraverted Toon CG as opposed to extraverted Realistic character is interesting and could indicate that extraversion was more appropriately expressed by the Toon CG character. 
Investigating render style across all personalities did not show many significant results, therefore we continue with the examination of individual personality effects.

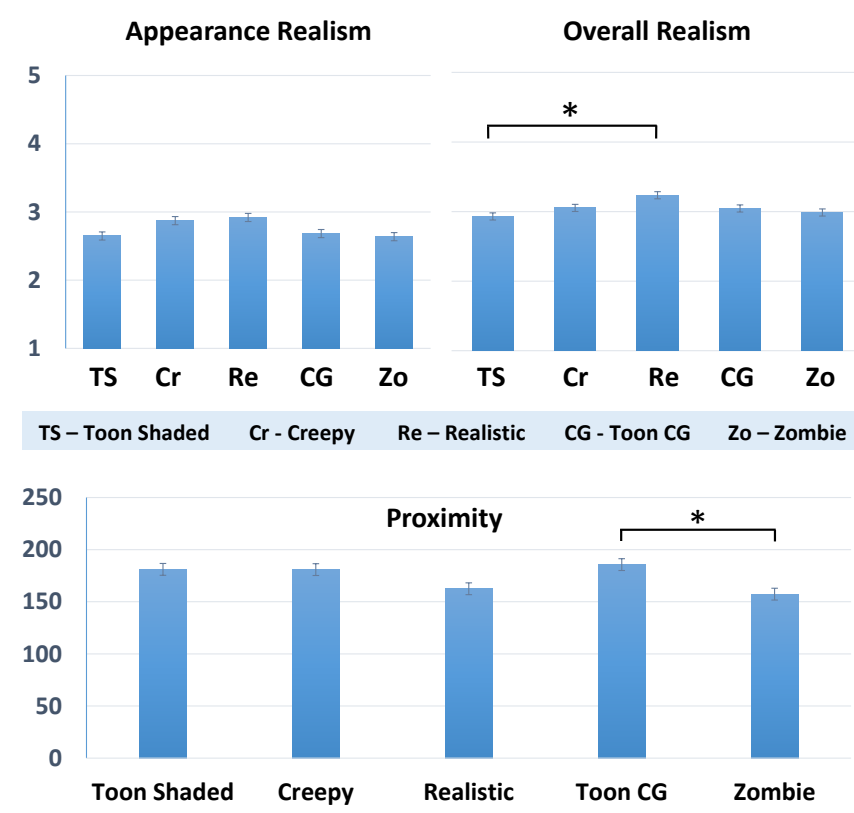

Fig. 5. Main effect of Render Style for two of the Realism items and Proximity. Star labeled lines point to significantly different means according to the post-hoc test $(*=p<0.05, * *=p<0.001)$. The Proximity distance shows the minimum distance from the character in centimeters. For example, we see that participants moved closer to the Zombie render style, especially compared to the Toon CG render style.

\section{2 b) The effect of Personality}

As seen in Figure 6, we found that the personality the character was exhibiting had an influence on our results, which was to be expected. The negative pole of the personalities (Introverted, Neurotic, Non Agreeable) induced more extreme scores for empathy, higher concern and unease, but also less excitement. Appeal was more positive also for the positive pole and eeriness was higher for the negative pole. As expected, some personalities were rated lower on motion realism, perhaps because these characters were moving less overall: emotionally stable character, rated higher on movement realism, had more body movement in comparison to the introverted one, which moved a lot less. The proximity measure, however, was not affected by personality. The character's personality therefore contributed to the variety of responses on empathy, affinity and even realism, but did not affect perceiver's behaviour.

While we did not find many effects of render style in Section a), by looking inside each personality and seeing the effect the render styles had on the participants' response, we find an indication that different render styles interact with the exhibited personality, which notably changes the response to that character (see Figures $1-4$ and Table 1 in the Supplementary Material). For example, the concern for a Realistic render style was high when he was portraying neurotic personality, but not when expressing non agreeable personality. Toon CG render style, on the other hand, improved co-presence when expressing the extraverted personality (in comparison to Realistic) and was less eerie in the agreeable one (more so than the Zombie render style). However, as with Realistic render style, this was not a pattern which repeated for every exhibited personality. The reasons behind this are difficult to determine from our experiment. For example, we see that the Realistic style is an appropriate choice if the intention is to reach a high level of concern for the neurotic situation but the style will make no difference if the character is expressing a non-agreeable personality. Also, when the Toon CG render style was expressing the extraverted personality, the ratings of co-presence were significantly higher, indicating perhaps that the combination of this render style and personality was a good design choice. We conclude that render style has a considerable effect on the viewer's response when the character is exhibiting personality traits.

Unexpectedly, proximity behaviour was not affected by personality. It is possible our situations which exhibit certain personalities did not vary enough in appeal and eeriness to really show responses on a behavioural level. Furthermore, the only significant effect for proximity we found for the agreeable personality, was conflicting. When paired with a character's appearance, the most eerie combination of style and personality as rated by the participants (Agreeable Zombie character) was also the character participants came closest to. This could mean that participants came closer to the character because of the fact it was eerie, possibly out of interest to examine mistakes. Proximity is slightly affected by the render style and personality combinations which might indicate interest to observe a particularly eerie character.
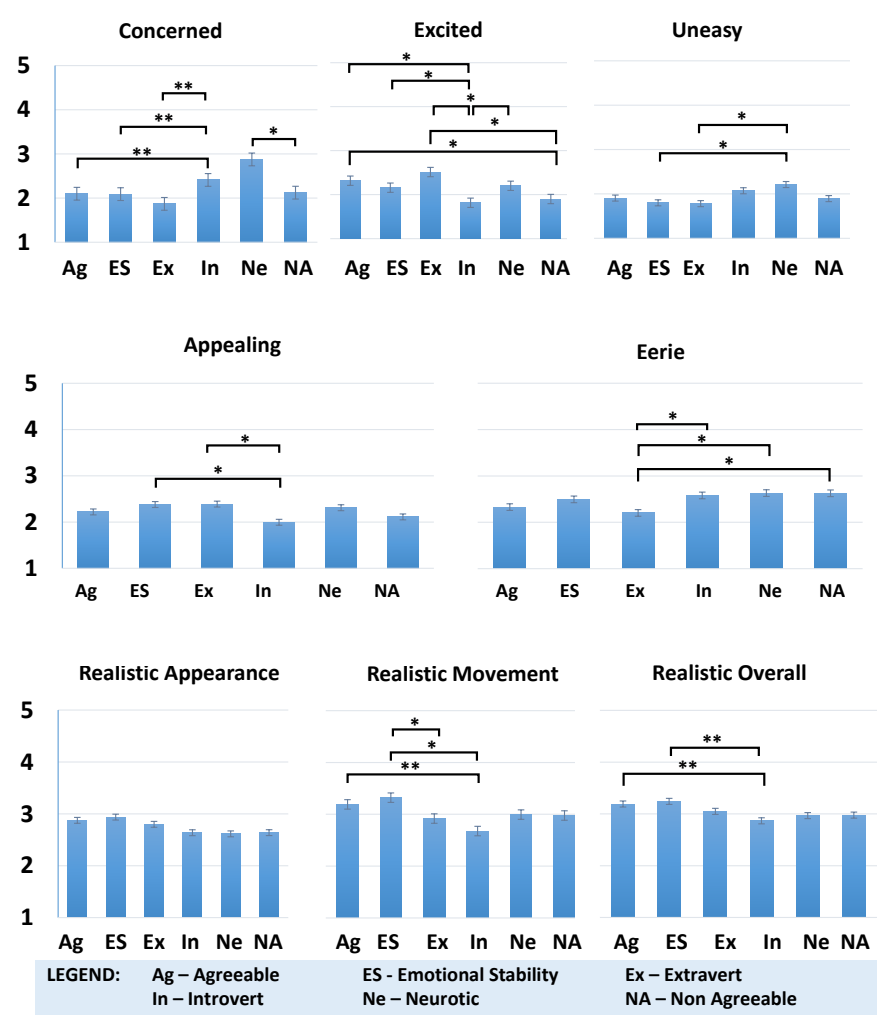

Fig. 6. Main effects of Personality. Star labeled lines point to significantly different means according to the post-hoc test $(*=p<0.05, * *=p<$ 0.001 ). While no effects of Personality on Familiarity, Proximity and Copresence were found, other dependent variables were affected. Individual comparisons between Personality types are found in Table ??.

\section{3 c) Attribution Bias}

We found Personality had a significant effect on Attribution $\left(\chi^{2}=\right.$ $88.71, d f=5, p=0.000$ ) and so did the Render Style (Figure 7, Table 2). We see that Extravert, Neurotic and Non Agreeable Personality were associated with the character's behaviour being attributed more to Situation than Character.

With further investigation, we found that certain render styles changed the attribution bias even further. The bottom graphs in Figure 7 show the significant differences which were obtained by comparing the effects of Render Style inside Personality. We found that Realistic 
render style significantly changed the attribution towards Situation in both Agreeable and Extravert conditions, while Creepy render style did so in the Neurotic condition. However, the most extreme and significant differences are only compared to certain render styles (Toon Shaded and Toon CG).

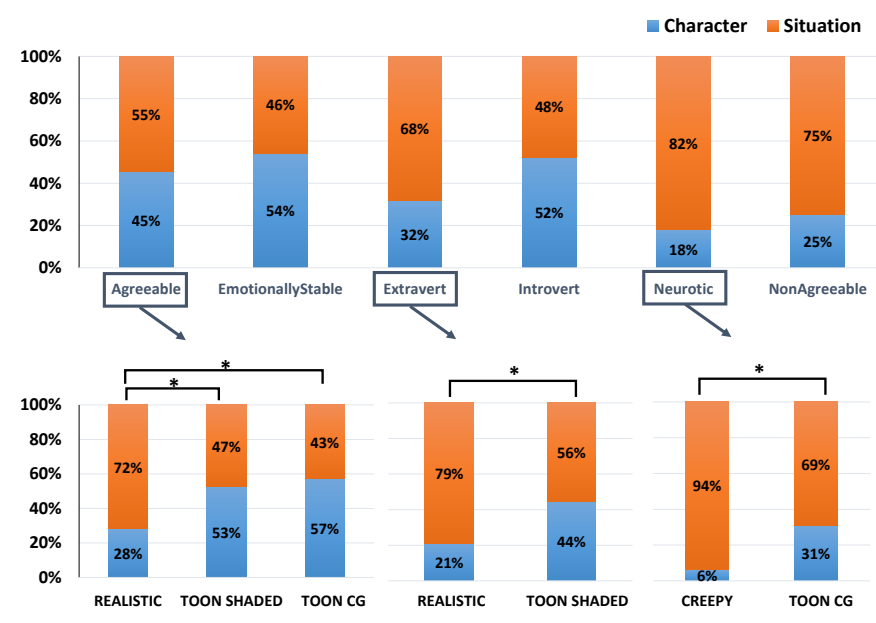

Fig. 7. Percentage of Attribution for Situation and Personality averaged over Render Style (top). Render style of the character significantly changes the attribution in the cases of Agreeable, Extraverted and Neurotic (bottom). Star labeled lines point to significantly different means according to the post-hoc test $(*=p<0.05, * *=p<0.001)$.

The main differences were therefore found for the render styles which were created to be more realistic (Realistic and Creepy) in comparison to other less realistic styles, which indicated that the character's behaviour was attributed more to the situation when the character was realistic. However, this happened only for particular personalities and not others. We included this test to explore automatic (or less automatic) processes towards realistic characters, but it is hard to conclude if realism was the source of difference in these results, since we did not achieve a high level of co-presence and the proximity results do not seem to indicate the behaviour of personal space which people exhibit in social situations. Therefore, it would not be strictly valid to speak about social cognitive effects of the attribution bias since other measures did not show our virtual characters induced socially cognitive processes. If our results were to be analysed in this fashion, they would indicate that Toon Shaded and Toon CG render styles were most difficult to process in certain situations since the attribution of a behaviour to situation was lower compared to other styles, but particularly to the realistic ones (Realistic and Creepy).

However, the fact that render style could change the attribution of behaviour at all, is an interesting result which prompts further investigation. Out of interest, we conducted a non-parametric Sperman's Rank Test correlation between Attribution and all the dependent variables. There were no significant correlations except a weak correlation with the variable Concerned, where higher concern was associated with attributing the character's behaviour to Situation $(r=0.08, p<0.05)$. This result, that higher concern for the character is associated with attributing his behaviour to a situation, could imply that empathy is higher for a character if we perceive him not being responsible for a difficult situation. Since this is further mediated by the character's appearance, it is possible that attribution bias could be used as a measure of empathy towards the character. But this can only be true for negative situations, since the attribution of behaviour to situation in positive context (such as our result for extraverted and agreeable personality) was not associated with higher excitement, for example. It could also be due to the lack of emotional scales or other measures of empathy, which would record more detailed emotional responses. We conclude that attribution bias is an interesting measure to investigate response to virtual characters but needs further exploration.

\section{4 d) Analysis of Demographics}

When exploring how participants' demographics affected our results, we found effects for Native on variables Concerned, Excited, Uneasy, Appeal, Realistic Appearance and Eerie, where non-native speakers were rating all scales significantly higher than the native speakers (see Supplementary Material, Table 2, for results). Game Experience had an effect on how participants rated Eerie, where passionate gamers where giving lower ratings of eeriness overall. Age made a difference in how participants were rating Appeal and Excited. The older the participants, the more excited they felt after watching the character but they also put lower markings for appeal. Other factors (Participant Sex, Character Experience) did not significantly influence our data.

Adding the demographic details in the analysis provided some interesting findings. For example, being a native speaker had a difference in most of our results measured with the questionnaire. This could possibly indicate differences in understanding the meaning of the words or cultural differences in perception, which were not the focus of our study but should be investigated further. Results such as game experience and age are interesting as well. Passionate gamers rated characters as less eerie, probably because they are more familiar with the limitations of the gaming platform and were more forgiving of mistakes on the characters. Older participants appeared to have enjoyed the experience more but the lower appeal ratings could indicate they would not be motivated to watch the character again. We conclude that some demographic information, such as age, country of origin and game experience of the participant should be taken into account when analysing responses to the virtual characters.

\section{Additional Experiment: Close-up}

Overall, our results provided us with some insights into how render style could affect our perception of the character in the virtual environment. However, the proximity measure did not give much indication of behavioural differences according to the factors. Furthermore, because of this measure, the participants had to be placed at an appropriate distance from the character, therefore small details in the facial animation were obscured. We decided to test if a closer view of the character would make a difference in how they rated him on the questionnaire items.

\subsection{Experiment Design}

This experiment was conducted in the last two weeks of the exhibition, therefore all the protocols regarding participants were the same. We tested 72 new participants ( 31 female, 41 male; average age: $26 \pm 12$ ). Every other aspect of the task also remained the same, only that the red spot was put approximately $1 \mathrm{~m}$ from the character on the very border of his collision sphere as opposed to having to observe the character $4 m$ away, as in the previous experiment (see Figure 8). Proximity had to be omitted from this experiment.
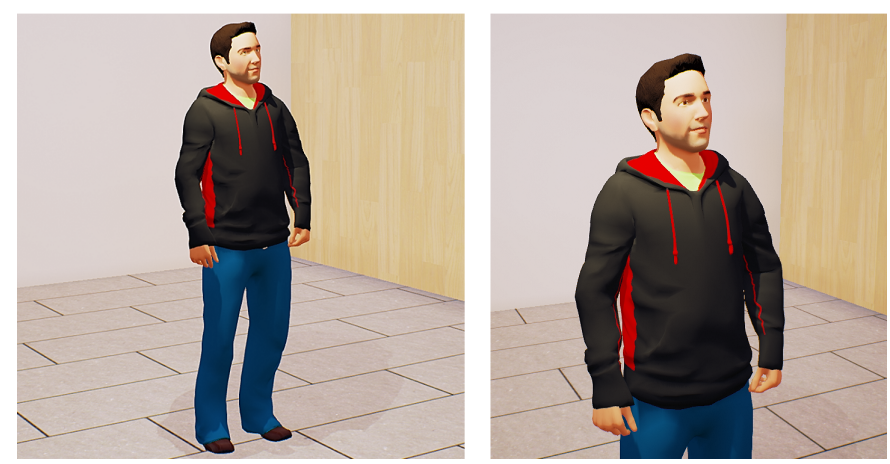

Fig. 8. Left: initial viewing distance for the character (Distance condition: Far). Right: the chosen distance for the Close-up experiment (Distance condition: Close); this distance was chosen so the character's face and hand gestures are visible. 
We changed the number of presented characters to get only the most relevant differences. We reduced the number of Render Style factors to 3 (Toon CG, Realistic, Zombie). We picked these three styles because results from the previous experiment gave the most prominent differences for these styles. Also, we reduced the number of personality types to 2 , one from the negative and one from the positive spectrum of the personality (Neurotic and Extravert) in order to get a matching number for both types of personality in our data set.

\subsection{Results}

In order to assess if the distance made a difference in how participants rated the characters, we examined differences between how participants rated the individual personalities (Extravert, Neurotic) and particular render style (Toon CG, Realistic, Zombie) on the dependent variables (Appeal, Realism, Empathy, Co-presence, Attribution) depending on the condition Distance (Close, Far). If data for the individual comparisons was not distributed normally, we analysed the differences by using Mann-Whitney U test, in case of normal distributions we chose independent sample $t$-test. For Attribution, as before, Pearson Chi-Squared test was used on the categorical data.

None of the comparisons between corresponding factors were different based on how close or far participants were standing, except for the Zombie render style expressing Neurotic personality. This character was rated more Eerie in the Close condition $(U(60)=4, Z=$ $-2.18, p=0.037)$. The Appearance Realism was also rated more realistic when participants were standing closer to the Neurotic Zombie character $(U(60)=11, Z=2.17, p=0.030)$.

Conclusion: the distance towards the character did not influence the subjective ratings except for the Neurotic Zombie character. The closer view in this condition increased the feeling of eeriness and appearance realism. Since no other differences were detected, we conclude overall, that the first chosen distance ( $4 m$ from the character) gave sufficient details of the character's appearance.

\section{General Discussion}

Our study analysed results of appearance and the role of personality in the context of an engaging, virtual reality experiment. We were interested to know, if realism could induce a negative response in viewers, similar to the uncanny valley hypothesis, or if a character's behavior is more detrimental to the perceived affinity in an immersive environment. Our results showed that it is actually a combination of both behavior and appearance.

Possibly the most striking result is that, overall, the render styles did not vary significantly in appeal, even though we purposely designed some characters to be unappealing and eerie. Render style only changed the appeal in combination with a particular personality, potentially a personality which was not "suitable" for a particular appearance. For example, realistic render style was found more appealing when the character in this style was portraying a neurotic personality. However, it was also seen as more eerie. A cartoon-looking character could have been a better choice for our extraverted personality, where the level of co-presence was found to be higher. Also, the Zombie render style, even though intentionally created to be eerie, was only seen as explicitly eerie when portraying an agreeable personality, possibly again since a positive personality would not be associated with such a character. Our results therefore show that the question of affinity towards virtual characters is complex and depends on the behavior, which supports similar findings of Bailenson et al. [4], where appearance realism was found to increase co-presence when the character's behaviour was realistic. Our behavioral data in a form of exhibited personality is more complex but still follows a particular logic - realistic render style increases empathy for characters in negative situations, whereas unappealing characters will not be associated with positive scenarios. There are some limitations to this conclusion. Namely, our results do not show a concise effect for all situations on all render styles. For example, it does not explain why the Zombie render style was particularly eerie only when expressing the Agreeable personality and not Extraverted or Emotionally stable. This could potentially be a limitation related to our constructed scenarios, which were designed to display personality traits. While careful consideration of creating scenarios based on observable personality traits from the existing literature was followed in our study, there are limitations to this approach. The reactions to the scenarios could have been influenced by the topic and not strictly to personality itself, e.g., people could associate with their own car being destroyed and the characters reaction to it would not seem neurotic, but rather quite reasonable. More experiments are needed to analyse which elements of the scenarios in particular contribute to the effects observed in our results and to what extent they could be attributed to the character's personality.

Some interesting relationships between dependent variables we used to study the effects were found as well. According to our results, Appeal was not related to lower eeriness. While eeriness and appeal represent two distinct categories as proposed by Ho and MacDorman [22], it is unlikely that an eerie character will also be appealing. This is an interesting finding and could indicate that a virtual character who exhibits a certain personality could be interesting to observe (appealing and engaging) while being eerie at the same time, as found for our zombie render style. For example, watching horror movies and particularly disturbing scenes in movies is scary but also entertaining. In addition, for most of the participants, virtual reality was a type of media which they hadn't experienced before. This could have affected our results by elevating the levels of appeal towards all the characters and perhaps disturb other assessments as well, such as proximity. If participants approached characters out of interest to observe details, proximity was not associated with higher co-presence with the character. Another explanation why zombie characters could be approached closely is associated with an artificial representation, which cannot be expected to exist in real life, therefore the threat is removed. A study of Bruneau et al. [7], exploring the effect of zombie vs. soldier characters in an immersive environment, found that walking through a crowd of characters was affected more when they were plausible in real life (soldiers), then when they were not (zombies). Both types of characters could be perceived as threatening, but only soldiers could be encountered in real life. To answer the question if it was implausibility or interest to observe details which made the participants come closer to zombie characters in our study, we would need to increase physical co-presence in our future work. This could be achieved by adding interactive cues, e.g., eye-gaze, as proposed by Bailenson et al. [3]. Another important aspect of interaction, which should be considered in the future, is selfrepresentation of the participant in the virtual environment. Seeing one's body in virtual reality would not only increase co-presence but also expand the behavioural measures to include analysis of nonverbal gestures as presented by Bailenson and Yee [5], to successfully measure appeal of the characters.

Another observed effect, which was interesting was that we did not get as many differences in appeal between personalities as in the study of Zibrek et al. [48], which might be due to the difference in experiment design, i.e., repeated measures as opposed to between group design, or perhaps because of different display platforms (screen-based vs. virtual reality). The only similar result was observed for introverted personality, which was rated significantly lower on appeal than other personality situations in both experiments. We conclude that experiment design should be carefully considered when measuring the response to virtual characters

The indirect measure of attribution proved to be an exciting new addition to the measurements as well, which extends the previous study of McDonnell [31] which used an indirect measure of trustworthiness but did not find an effect of render style. Our explanation was that the lie detection task in that study was too difficult for the participants and therefore the response showed more cognitive effort than perceptual effects. Even though it is hard to explain where the results from our study comes from and if it actually indicates the ease of processing, similarly to the concept developed by Gilbert [17], it showed some differences according to which render style is exhibiting a certain personality and the attribution of behaviour towards situation seems to be associated with empathy as well. This is not unexpected, since higher empathy is evoked when we feel the person is not responsible for the negative outcome but was subjected to a difficult situation. However, 
based on our experiment, stronger conclusions cannot be made on this topic.

When observing humans, we make particular stereotyped judgments, which were not included in our design. We only examined the effects of render style and personality scenarios performed by one male model. We are aware that our conclusions are thus limited, since there are studies showing the effect of character gender on the behaviour and subjective responses after virtual reality exposures. The study of Fox and Bailenson [14] not only explored stereotyped behaviour and response to female characters but also considered the clothes to be an important social signal - something which we did not consider. Besides gender, studies show importance of social status [1] and race [21]. These were not aspects we included in the design of our experiments but are important when examining social constructs in virtual reality. Further analysis, including more characters, is needed to estimate their perception in complex social encounters. However, we believe that our study effectively examined visual realism and used scenarios, which are both complex and controlled.

We conclude that this study provides sufficient evidence that realism by itself is not enough to explain the changes in appeal when observing characters in virtual reality, since character's appearance interacts with its behaviour, in our case the exhibited personality. Exactly which types of personality traits are more appealing when on characters of particular appearance type needs further testing. We found a positive association between realistic style and a personality, which raised the levels of empathy. Purposefully eerie styles did not fit certain positive poles of personality. These findings could be considered when designing virtual characters.

\section{ACKNOWLEDGEMENTS}

This research was funded by the Science Foundation Ireland as part of the "Game Face" project (grant 13/CDA/2135) and ADAPT Centre for Digital Content Technology (grant 13/RC/2106) projects.

\section{REFERENCES}

[1] J. N. Bailenson, E. Aharoni, A. C. Beall, R. E. Guadagno, A. Dimov, and J. Blascovich. Comparing behavioral and self-report measures of embodied agents' social presence in immersive virtual environments. In 7th Annual International Workshop on Presence, Valencia, Spain, 2004.

[2] J. N. Bailenson, J. Blascovich, A. C. Beall, and J. M. Loomis. Equilibrium theory revisited: Mutual gaze and personal space in virtual environments Presence, 10(6):583-598, 2001.

[3] J. N. Bailenson, J. Blascovich, A. C. Beall, and J. M. Loomis. Interpersonal distance in immersive virtual environments. Personality and Social Psychology Bulletin, 29(7):819-833, 2003.

[4] J. N. Bailenson, K. R. Swinth, C. L. Hoyt, S. Persky, A. Dimov, and J. Blascovich. The independent and interactive effects of embodied-agent appearance and behavior on self-report, cognitive, and behavioral markers of copresence in immersive virtual environments. Presence, 14(4):379393, 2005.

[5] J. N. Bailenson and N. Yee. Digital chameleons: Automatic assimilation of nonverbal gestures in immersive virtual environments. Psychological science, 16(10):814-819, 2005.

[6] J. Blascovich. Social influence within immersive virtual environments. In The social life of avatars, pp. 127-145. Springer, 2002.

[7] J. Bruneau, A.-H. Olivier, and J. Pettré. Going through, going around: a study on individual avoidance of groups. IEEE transactions on visualization and computer graphics, 21(4):520-528, 2015.

[8] E. Carter, M. Mahler, and J. Hodgins. Unpleasantness of animated characters increases viewer attention to faces. In Proceedings of the ACM Symposium in Applied Perception, pp. 35-40, 2013.

[9] T. Chaminade, J. Hodgins, and M. Kawato. Anthropomorphism influences perception of computer-animated characters' actions. Social Cognitive and Affective Neuroscience, 2(3):206-216, 2007.

[10] M. Cheetham and L. Jancke. Perceptual and category processing of the uncanny valley hypothesis' dimension of human likeness: some methodological issues. JoVE (Journal of Visualized Experiments), (76):e4375-e4375, 2013.

[11] P. T. Costa and R. R. McCrae. Professional manual: revised neo personality inventory (NEO-PI-R) and neo five-factor inventory (NEO-FFI). Odessa, FL: Psychological Assessment Resources, 1992.
[12] M. H. Davis. Measuring individual differences in empathy: Evidence for a multidimensional approach. Journal of personality and social psychology, 44(1):113, 1983.

[13] J.-M. Dewaele and A. Furnham. Personality and speech production: a pilot study of second language learners. Personality and Individual Differences, 28(2):355-365, 2000.

[14] J. Fox and J. N. Bailenson. Virtual virgins and vamps: The effects of exposure to female characters' sexualized appearance and gaze in an immersive virtual environment. Sex Roles, 61(3):147-157, 2009.

[15] M. Garau, M. Slater, V. Vinayagamoorthy, A. Brogni, A. Steed, and M. A. Sasse. The impact of avatar realism and eye gaze control on perceived quality of communication in a shared immersive virtual environment. In Proceedings of the SIGCHI conference on Human factors in computing systems, pp. 529-536. ACM, 2003.

[16] D. T. Gilbert. Ordinary personology. The Handbook of Social Psychology, 2:89-150, 1998.

[17] D. T. Gilbert, B. W. Pelham, and D. S. Krull. On cognitive busyness: When person perceivers meet persons perceived. Journal of Personality and Social Psychology, 54(5):733, 1988.

[18] A. Gill and J. Oberlander. Perception of e-mail personality at zeroacquaintance: Extraversion takes care of itself; neuroticism is a worry. In Proceedings of the 25th Annual Conference of the Cognitive Science Society, pp. 456-461, 2003.

[19] B.-C. S. Golan, Ofer and J. Hill. The cambridge mindreading (cam) face-voice battery: Testing complex emotion recognition in adults with and without asperger syndrome. Journal of Autism and Developmental Disorders, 36(2):169-183, 2006.

[20] L. R. Goldberg. An alternative "description of personality": the big-five factor structure. Journal of personality and social psychology, 59(6):1216, 1990.

[21] V. Groom, J. N. Bailenson, and C. Nass. The influence of racial embodiment on racial bias in immersive virtual environments. Social Influence, 4(3):231-248, 2009.

[22] C.-C. Ho and K. F. MacDorman. Revisiting the uncanny valley theory: Developing and validating an alternative to the godspeed indices. Computers in Human Behavior, 26(6):1508-1518, 2010.

[23] J. Hodgins, S. Jörg, C. O'Sullivan, S. I. Park, and M. Mahler. The saliency of anomalies in animated human characters. ACM Transactions on Applied Perception, 7(4), 2010.

[24] J. Hyde, E. Carter, S. Kiesler, and J. Hodgins. Perceptual effects of damped and exaggerated facial motion in animated characters. In Proceedings of the 10th IEEE International Conference on Automatic Face and Gesture Recognition, pp. 1-6, 2013.

[25] G. Johansson. Visual perception of biological motion and a model for its analysis. Perception \& Psychophysics, 14(2):201-211, 1973.

[26] O. P. John, L. P. Naumann, and C. J. Soto. Paradigm shift to the integrative big five trait taxonomy. Handbook of personality: Theory and research, $3: 114-158,2008$.

[27] J. Kätsyri, K. Förger, M. Mäkäräinen, and T. Takala. A review of empirical evidence on different uncanny valley hypotheses: support for perceptual mismatch as one road to the valley of eeriness. Frontiers in Psychology, 6, 2015.

[28] K. C. Klauer, A. Voss, and C. Stahl. Cognitive methods in social psychology. Guilford Press, 2012.

[29] R. M. Latta. Relation of status incongruence to personal space. Personality and Social Psychology Bulletin, 4(1):143-146, 1978.

[30] K. F. MacDorman, R. D. Green, C.-C. Ho, and C. T. Koch. Too real for comfort? uncanny responses to computer generated faces. Computers in human behavior, 25(3):695-710, 2009.

[31] R. McDonnell, M. Breidt, and H. Buelthoff. Render me real Investigating the effect of render style on the perception of animated virtual humans. ACM Transactions on Graphics, 31(4):91:1-91:11, 2012.

[32] M. R. Mehl, S. D. Gosling, and J. W. Pennebaker. Personality in its natural habitat: manifestations and implicit folk theories of personality in daily life. Journal of personality and social psychology, 90(5):862, 2006.

[33] M. Mori. The uncanny valley. Energy, 7(4):33-35, 1970.

[34] J. Musek. A general factor of personality: Evidence for the big one in the five-factor model. Journal of Research in Personality, 41(6):1213-1233, 2007.

[35] J. A. Nelder and R. J. Baker. Generalized linear models. Encyclopedia of Statistical Sciences, 1972.

[36] K. Nowak. The influence of anthropomorphism on social judgment in social virtual environments. In Annual Convention of the International 
Communication Association, Washington, DC, 2001.

[37] L. Ring, D. Utami, and T. Bickmore. The right agent for the job? In International Conference on Intelligent Virtual Agents, pp. 374-384. Springer, 2014.

[38] L. Ross. The intuitive psychologist and his shortcomings: Distortions in the attribution process. Advances in Experimental Social Psychology, 10:173-220, 1977.

[39] J. Seyama and R. S. Nagayama. The uncanny valley: Effect of realism on the impression of artificial human faces. Presence: Teleoperators and Virtual Environments, 16(4):337-351, 2007.

[40] M. Slater and A. Steed. Meeting people virtually: Experiments in shared virtual environments. In The Social Life of Avatars, pp. 146-171. Springer, 2002.

[41] A. Tinwell, D. A. Nabi, and J. P. Charlton. Perception of psychopathy and the uncanny valley in virtual characters. Computers in Human Behavior, 29(4):1617-1625, 2013.

[42] M. Volante, S. V. Babu, H. Chaturvedi, N. Newsome, E. Ebrahimi, T. Roy, S. B. Daily, and T. Fasolino. Effects of virtual human appearance fidelity on emotion contagion in affective inter-personal simulations. IEEE transactions on visualization and computer graphics, 22(4):1326-1335, 2016.

[43] C. Wallraven, H. H. Bülthoff, D. W. Cunningham, J. Fischer, and D. Bartz. Evaluation of real-world and computer-generated stylized facial expressions. ACM Transactions on Applied Perception, 4(3), 2007.

[44] D. Watson, L. A. Clark, and A. Tellegen. Development and validation of brief measures of positive and negative affect: the panas scales. Journal of personality and social psychology, 54(6):1063, 1988.

[45] Y. Yamada, T. Kawabe, and K. Ihaya. Categorization difficulty is associated with negative evaluation in the "uncanny valley" phenomenon. Japanese Psychological Research, 55(1):20-32, 2013.

[46] E. Zell, C. Aliaga, A. Jarabo, K. Zibrek, D. Gutierrez, R. McDonnell, and M. Botsch. To stylize or not to stylize?: The effect of shape and material stylization on the perception of computer-generated faces. ACM Transactions on Graphics, 34(6):184:1-184:12, 2015.

[47] K. Zibrek, E. Kokkinara, and R. McDonnell. Don't stand so close to me: investigating the effect of control on the appeal of virtual humans using immersion and a proximity-based behavioral task. In Proceedings of the ACM Symposium on Applied Perception, p. 3. ACM, 2017.

[48] K. Zibrek and R. McDonnell. Does render style affect perception of personality in virtual humans? In Proceedings of the ACM Symposium on Applied Perception, pp. 111-115. ACM, 2014. 